\title{
Effects of minocycline on macrolide-unresponsive Mycoplasma pneumoniae pneumonia in children: a single-center retrospective study
}

\author{
Jiande Chen", Xinyi Qi", Yong Yin, Lei Zhang, Jing Zhang, Shuhua Yuan \\ Department of Respiratory Medicine, Shanghai Children's Medical Center, School of Medicine, Shanghai Jiao Tong University, Shanghai, China \\ Contributions: (I) Conception and design: J Chen, X Qi, Y Yin; (II) Administrative support: Y Yin, L Zhang; (III) Provision of study materials or \\ patients: Y Yin, L Zhang, J Zhang, S Yuan; (IV) Collection and assembly of data: J Chen, X Qi; (V) Data analysis and interpretation: All authors; (VI) \\ Manuscript writing: All authors; (VII) Final approval of manuscript: All authors. \\ "These authors contributed equally to this work. \\ Correspondence to: Yong Yin. Department of Respiratory Medicine, Shanghai Children's Medical Center, School of Medicine, Shanghai Jiao Tong \\ University, No.1678 Dongfang Road, Pudong, Shanghai 200127, China. Email: yinyong9999@163.com.
}

Background: Macrolide-resistant Mycoplasma pneumoniae $(M p)$ has become widespread in the world. We sought to determine the independently associated risk factors for refractory $M p$ pneumonia among macrolide-unresponsive $M p$ pneumonia children treated with minocycline and to investigate the effects of minocycline against macrolide-unresponsive $M p$ pneumonia.

Methods: In our center, we retrospectively analyzed the data of hospitalized macrolide-unresponsive $M p$ pneumonia patients aged $\leq 18$ years old who changed macrolide therapies to minocycline treatments between March 2013 and September 2018. Patient characteristics and defervescence after minocycline treatment were compared between refractory $M p$ pneumonia and non-refractory $M p$ pneumonia groups. Multivariable logistic regression analysis was performed among these macrolide-unresponsive $M p$ pneumonia patients.

Results: Among 150 included macrolide-unresponsive $M p$ pneumonia children treated with minocycline; 30 cases $(20.0 \%)$ were refractory $M p$ pneumonia. Duration of macrolide treatment before administration of minocycline (odds ratio $=2.87,95 \% \mathrm{CI}: 1.79-4.61, \mathrm{P}<0.001$ ) and serum procalcitonin levels (odds ratio $=13.50,95 \%$ CI: $1.22-149.57, \mathrm{P}=0.034)$ were independently associated with refractory $M p$ pneumonia. Defervescence after minocycline treatment was significantly longer among the refractory $M p$ pneumonia group than in the non-refractory $M p$ pneumonia group (median 2 vs. 1 day, $\mathrm{P}<0.001)$. Only one case $(0.7 \%$ ) suspected of a side effect of minocycline therapy was observed.

Conclusions: Two risk factors independently associated with refractory $M p$ pneumonia were determined. Early use of minocycline might safely prevent macrolide-unresponsive $M p$ pneumonia from progressing to refractory $M p$ pneumonia.

Keywords: Mycoplasma pneumoniae (Mp); macrolide; minocycline; defervescence

Submitted Aug 02, 2021. Accepted for publication Aug 15, 2021.

doi: $10.21037 / \mathrm{tp}-21-356$

View this article at: https://dx.doi.org/10.21037/tp-21-356

\section{Introduction}

Mycoplasma pneumoniae $(M p)$ is a common causative pathogen of community-acquired pneumonia (CAP) in children. Macrolides are the first line antimicrobials against $M p$ infections in the clinical practice. However, macrolide- resistant $M p$ has become widespread in the world (1-4). A large variance has been identified in the macrolide-resistant $M p$ rates (54.5-100\%) across different regions of China (5). In Shanghai, the macrolide-resistant $M p$ rate has been estimated to be $90 \%$ (6). The clinical and bacteriological 
efficacy of macrolides for treating macrolide-resistant $M p$ infection was lower than that for children of macrolidesensitive $M p$ infection $(7,8)$. Besides an increasing bacterial load, an excessive host immune response among patients with macrolide-unresponsive $M p$ might be the pathogenesis of the lung injury $(9,10)$. Without properly treating these patients, refractory Mycoplasma pneumoniae pneumonia (RMPP) may occur.

It's well known that minocycline (tetracycline) is effective in the treatment of children with macrolideresistant $M p$ infection $(7,8,11)$. In the Japanese guidelines for the management of respiratory infectious diseases in children, changing treatment to a tetracycline antibiotic has been recommended in possible macrolide-resistant $M p$ infection (12). The purpose of this study was to determine the independently associated risk factors for RMPP among macrolide-unresponsive Mycoplasma pneumoniae pneumonia (MUMPP) children treated with minocycline and to investigate the effects of minocycline against MUMPP. We present the following article in accordance with the STROBE reporting checklist (available at https://dx.doi. org/10.21037/tp-21-356).

\section{Methods}

\section{Subjects and study design}

We retrospectively analyzed the medical records of patients with MUMPP aged $\leq 18$ years old who changed macrolide therapies to minocycline treatments and were hospitalized at Shanghai Children's Medical Center between March 2013 and September 2018.

The diagnosis of Mycoplasma pneumoniae pneumonia (MPP) was defined as: (I) CAP was confirmed: the presence of fever, acute respiratory symptoms (cough, tachypnoea, difficult breathing), or both, plus presence of new infiltrate on chest radiography or consolidation (13); (II) $M p$ infection was confirmed: positive detection of $M p$ RNA in throat swab samples or/and $M_{p}$ DNA in sputum samples.

Among MPP patients, MUMPP was defined as persistent fever $\geq 38.0{ }^{\circ} \mathrm{C}$ at $\geq 72$ hours after macrolide treatment (14). The MUMPP cases were divided into two groups, RMPP and non-refractory Mycoplasma pneumoniae pneumonia (NRMPP).

RMPP was defined as cases showing persistent fever and clinical as well as radiological deterioration despite macrolide therapy for 7 days or more (15).

Patients whose minocycline were: (I) used due to macrolide allergy; (II) misused; (III) used in macrolideresponsive MPP; (IV) used to treat non-Mp infection; or (V) used before MUMPP was confirmed were excluded.

According to the requirements of our center, informed consent was obtained from their guardians before minocycline therapy. Minocycline was administered orally twice daily at a dose of $1-2 \mathrm{mg} / \mathrm{kg} / \mathrm{time}$ for 7-10 days. Because of side effects such as tooth discoloration, minocycline was mainly chosen for patients $\geq 8$ years old.

The study was conducted in accordance with the Declaration of Helsinki (as revised in 2013). The ethical committee of Shanghai Children's Medical Center approved the study (No. SCMCIRB-W2021035) and individual consent for this retrospective analysis was waived.

\section{Data collection}

The collected data from the medical records included clinical features, laboratory data, chest X-ray findings, treatments, hospital stays, time to defervescence after initial minocycline treatment, and side effects associated with minocycline one week after discharge. Extrapulmonary symptoms included pericarditis or myocarditis, skin rash, hepatitis, arthralgia, anemia or thrombocytopenia, and neurologic symptoms. Coinfection with other respiratory pathogens was defined as having documented bacterial (obtained by culture of respiratory secretions), viral (identified through MultiResPathogen Nucleic Acid Assay (16) for respiratory syncytial virus, adenovirus, parainfluenza virus, and influenza virus $A$ and $B$ ), or fungal infection (determined by metagenomic analysis of bronchoalveolar lavage fluid) and $M p$ infection concurrently within the same disease event. The chest X-ray findings were from the records read by two radiologists and classified as interstitial pneumonia, lobar pneumonia, or bronchopneumonia. Interstitial pneumonia was characterized by reticular and irregular opacities with increased perivascular makings presenting in interstitial infiltrate. Lobar pneumonia and bronchopneumonia were defined as the presence of a homogeneous airspace consolidation and an inhomogeneous airspace consolidation, respectively (17).

\section{Mp RNA detection}

Throat swabs were obtained within $24 \mathrm{~h}$ of admission. A qualitative diagnostic kit (Wuhan Zhongzhi Biotechnologies Inc., Hubei, China) for $M p$ RNA was used to determine 
$M p$ infection according to methods published before (16). In brief, pathogen nucleic acids were reverse transcribed to cDNA. Amplified RNA products were acquired from cDNA using T7 RNA polymerase. Fluorescence signals generated from the chemiluminescence mixture of RNA amplification products were then detected.

\section{Mp DNA detection}

The qualitative determination of $M p$ DNA that may be present in a sputum sample was detected using Mycoplasma Pneumoniae Real Time PCR Kit (Liferiver Bio-Tech Corp., Shanghai, China). Briefly, DNA was extracted from a sputum sample. Mp DNA was specifically amplified by the master containing reagents and enzymes. The amplified $\mathrm{Mp}$ DNA fragment was detected by fluorimeter channel FAM with the fluorescent quencher BHQ1.

\section{Statistical analysis}

Data were presented as medians and interquartile range for continuous variables and frequencies and percentages for categorical variables. Continuous variable comparisons between the groups were performed using Mann-Whitney $U$ test. Categorical variables were compared using the $\chi^{2}$ test or Fisher exact test. For investigating the independent risk factors of RMPP among MUMPP patients treated with minocycline, the significance of each variable in Table 1 was assessed by univariable logistic regression analysis. Variables with a $\mathrm{P}$ value $<0.05$ were included in the multivariable logistic regression analysis. Statistical significance was determined as a 2 -sided $\mathrm{P}$ value $<0.05$. Statistical analyses were performed using SPSS software 25.0 (IBM SPSS Statistics, Armonk, NY, USA).

\section{Results}

\section{Patient characteristics}

A total of 163 CAP patients treated with minocycline were screened; of those, 150 children with MUMPP whose macrolide therapies were changed to minocycline treatments were included in this study (Figure 1). Among these included patients, 30 cases $(20.0 \%)$ were in the RMPP group, and 120 (80.0\%) were in the NRMPP group. Patient characteristics were summarized in Table 1. Among the RMPP group, two patients were coinfected with bacteria (including Streptococcus pneumoniae and
Pseudomonas aeruginosa), three patients were coinfected with viruses (including respiratory syncytial virus, adenovirus, and influenza virus $B$ ), and one patient was coinfected with fungus (Candida albicans). Among the NRMPP group, three patients were coinfected with bacteria (including Klebsiella pneumonia, enterobacter cloacae, and Acinetobacter baumannii), four patients were coinfected with viruses (including respiratory syncytial virus, adenovirus, parainfluenza virus, and influenza virus A), and no patient was coinfected with fungus. Among the MUMPP patients treated with minocycline, the state of illness was more serious in the RMPP group than in the NRMPP group (Table 2).

\section{Risk factors for RMPP among MUMPP patients treated with minocycline}

Multivariable logistic regression analysis was performed to determine the independently associated risk factors for RMPP among MUMPP patients treated with minocycline. As Table 3 shows, duration of macrolide treatment before administration of minocycline (odds ratio $=2.87,95 \%$ CI: 1.79-4.61, $\mathrm{P}<0.001)$ and serum procalcitonin levels (odds ratio $=13.50,95 \%$ CI: $1.22-149.57, \mathrm{P}=0.034$ ) were independently associated with increased odds of RMPP.

\section{Defervescence after minocycline treatment}

After the change to minocycline treatments among those MUMPP patients, time to defervescence ranged from 1 to 16 days with a median of 1 day (interquartile range 1-2). After stratifying, time to defervescence after minocycline treatment was significantly longer among the RMPP group than in the NRMPP group (median 2 vs. 1 day, $\mathrm{P}<0.001$ ).

\section{Safety}

One week after discharge, only one case $(0.7 \%)$ with increased serum levels of alanine aminotransferase (ALT), which quickly returned to normal value after discontinuation of medication, was suspected to be caused by minocycline treatment.

\section{Discussion}

To our knowledge, this is the first study to determine the independently associated risk factors for RMPP among MUMPP children treated with minocycline. Then, we 
Table 1 Patient characteristics

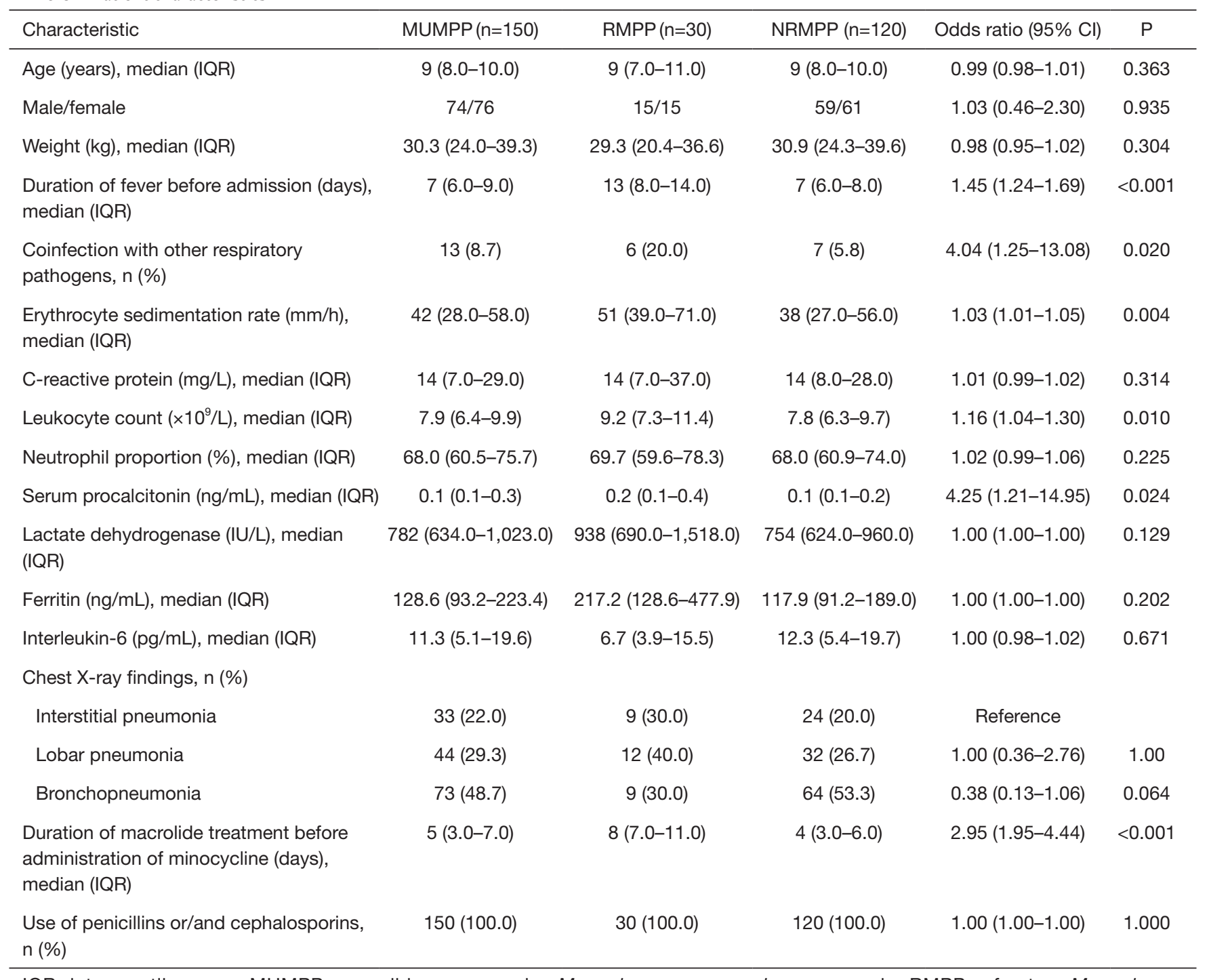

IQR, interquartile range; MUMPP, macrolide-unresponsive Mycoplasma pneumoniae pneumonia; RMPP, refractory Mycoplasma pneumoniae pneumonia; NRMPP, non-refractory Mycoplasma pneumoniae pneumonia; $\mathrm{Cl}$, confidence interval.

investigated the effects of minocycline against MUMPP.

The progression of MUMPP to RMPP led to serious clinical adverse consequences. When compared with NRMPP patents, RMPP ones required more frequent use of corticosteroid and bronohoalveolarlavage, were more likely to be admitted to ICU, had a longer length of hospital stay, and might be associated with more extrapulmonary complications (Table 2). Preventing MUMPP from progressing to RMPP may reduce the severity of illness.

Besides the occurrence of resistant strains, excessive inflammatory responses play a vital role in the pathogenesis of RMPP (9). Inflammatory regulatory factors such as high-mobility group box protein 1 (9) and microRNA-146a-5p (18) have been proved to be actively involved in regulating inflammation of RMPP and serve as molecular markers for RMPP. Coinfections (19) and corticosteroid resistance (20) may also contribute to the development of RMPP. Identifying the risk factors of RMPP will be of great significance for preventing the occurrence of RMPP.

Duration of macrolide treatment before administration of minocycline was independently associated with increased odds of RMPP. This indicates that early use of minocycline might prevent MUMPP from progressing to RMPP. In 




Figure 1 Flowchart of included patients.

Table 2 Severity of MUMPP treated with minocycline

\begin{tabular}{|c|c|c|c|c|}
\hline Characteristic & MUMPP (n=150) & $\operatorname{RMPP}(n=30)$ & NRMPP $(n=120)$ & $\mathrm{P}$ \\
\hline Patients requiring bronohoalveolarlavage, $\mathrm{n}(\%)$ & $44(29.3)$ & $21(70.0)$ & $23(19.2)$ & $<0.001$ \\
\hline Patients requiring ICU admission, $\mathrm{n}(\%)$ & $4(2.7)$ & $4(13.3)$ & $0(0.0)$ & 0.001 \\
\hline Duration of hospital stay, median days (IQR) & $7(5.0-9.0)$ & $12(9.0-17.0)$ & $6(5.0-8.0)$ & $<0.001$ \\
\hline
\end{tabular}

IQR, interquartile range; MUMPP, macrolide-unresponsive Mycoplasma pneumoniae pneumonia; RMPP, refractory Mycoplasma pneumoniae pneumonia; NRMPP, non-refractory Mycoplasma pneumoniae pneumonia; ICU, intensive care unit.

Table 3 Variables associated with RMPP among MUMPP children treated with minocycline in multivariable logistic regression analysis

\begin{tabular}{lcc}
\hline Variable & OR $(95 \% \mathrm{Cl})$ & $\mathrm{P}$ value \\
\hline Duration of fever before admission & $1.21(0.98-1.50)$ & 0.080 \\
Coinfection with other respiratory pathogens & $1.66(0.14-19.35)$ & 0.686 \\
Erythrocyte sedimentation rate & $0.99(0.95-1.03)$ \\
Leukocyte count & $1.18(0.96-1.46)$ & 0.535 \\
Serum procalcitonin & $13.50(1.22-149.57)$ & 0.121 \\
Duration of macrolide treatment before administration of minocycline & $2.87(1.79-4.61)$ & 0.034 \\
\hline
\end{tabular}

MUMPP, macrolide-unresponsive Mycoplasma pneumoniae pneumonia; RMPP, refractory Mycoplasma pneumoniae pneumonia; OR, odds ratio; $\mathrm{Cl}$, confidence interval.

the Japanese guidelines for the management of respiratory infectious diseases in children, changing treatment to a tetracycline antibiotic has been recommended in a lack of defervescence within $48 \mathrm{~h}$ after the initiation of macrolide therapy (12). We recommend changing the antibiotic treatment to minocycline at $72 \mathrm{~h}$ when MUMPP is suspected, especially in settings without possibility of testing mutations in the $23 \mathrm{~S}$ ribosomal RNA gene.

Minocycline has excellent bactericidal activity against macrolide-unresponsive $\mathrm{Mp}$ and macrolide-susceptible $\mathrm{Mp}$ $(7,21)$. In China, high macrolide resistance $(54.5-100 \%)$ has been observed across different regions $(5,6,22)$. 
Early application of minocycline may effectively reduce the macrolide-unresponsive Mp burden. In addition, minocycline has been considered beneficial for diseases with an inflammatory basis (23). Therefore, minocycline may exert anti-inflammatory activities that are independent of its bactericidal activity among MUMPP patients.

Increased PCT levels (24) are common in RMPP patients. In our study, serum procalcitonin levels were independently associated with increased odds of RMPP among MUMPP patients treated with minocycline. While patients with normal value of procalcitonin might be considered to be a low-risk subgroup of RMPP, the incidence of RMPP would increase as the serum procalcitonin levels increased. Increased levels of serum procalcitonin might be an indicator of early initiation of minocycline therapy.

Consistent with other studies $(7,8,11,25,26)$, minocycline showed good clinical efficacy in our study. Defervescence with a median of 1 day (interquartile range 1-2) was observed in all MUMPP patients after minocycline therapy. However, when MUMPP progressed to RMPP, time to defervescence after minocycline treatment became longer (median 2 vs. 1 day, $\mathrm{P}<0.001$ ). This further indicates the necessity of early application of minocycline in MUMPP patients.

When used in pediatric patients, minocycline rarely may cause side effects $(8,27)$. In our study, only one case $(0.7 \%)$ with increased serum levels of ALT was suspected to be caused by minocycline. Minocycline might be very safe in treating pediatric patients with MUMPP.

One limitation is that our study was not a prospective study. Therefore, selective bias could not be avoided. Randomized therapeutic trials are necessary to further support our findings. Because drug resistance gene detection is not a routine test item in our center, no performance in detecting mutations in the $23 \mathrm{~S}$ ribosomal RNA gene which is associated with macrolide-resistant $\mathrm{Mp}$ is another limitation. However, if MUMPP can be effectively treated without drug resistance gene test results, this minocycline therapy might have better economic and clinical benefits.

\section{Conclusions}

Duration of macrolide treatment before administration of minocycline and serum PCT levels are independent risk factors for RMPP among MUMPP pediatric patients treated with minocycline. Early use of minocycline might safely prevent MUMPP from progressing to RMPP.

\section{Acknowledgments}

We acknowledge the support of the study on the prevalence of Mycoplasma pneumoniae infection and macrolides resistance in outpatient and hospitalized children with acute respiratory illness in China (Shanghai Children's Medical Center). We thank Nalisa Lubasi David for his support in language editing.

Funding: This work was supported by the Scientific research project of Shanghai Pudong New Area Health Committee (No. PW2017E-1), the Scientific research project of Shanghai Pudong New Area Health Committee (No. PW2019A-37), and the Scientific research projects of Shanghai Science and Technology Commission (No. 19441909000).

\section{Footnote}

Reporting Checklist: The authors have completed the STROBE reporting checklist. Available at https://dx.doi. org/10.21037/tp-21-356

Data Sharing Statement: Available at https://dx.doi. org/10.21037/tp-21-356

Conflicts of Interest: All authors have completed the ICMJE uniform disclosure form (Available at https://dx.doi. org/10.21037/tp-21-356). The authors have no conflicts of interest to declare.

Ethical Statement: The authors are accountable for all aspects of the work in ensuring that questions related to the accuracy or integrity of any part of the work are appropriately investigated and resolved. The study was conducted in accordance with the Declaration of Helsinki (as revised in 2013). The study was approved by the ethical committee of Shanghai Children's Medical Center (No. SCMCIRB-W2021035) and individual consent for this retrospective analysis was waived.

Open Access Statement: This is an Open Access article distributed in accordance with the Creative Commons Attribution-NonCommercial-NoDerivs 4.0 International License (CC BY-NC-ND 4.0), which permits the noncommercial replication and distribution of the article with the strict proviso that no changes or edits are made and the original work is properly cited (including links to both the formal publication through the relevant DOI and the license). 
See: https://creativecommons.org/licenses/by-nc-nd/4.0/.

\section{References}

1. Qu J, Chen S, Bao F, et al. Molecular characterization and analysis of Mycoplasma pneumoniae among patients of all ages with community-acquired pneumonia during an epidemic in China. Int J Infect Dis 2019;83:26-31.

2. Morozumi M, Iwata S, Hasegawa K, et al. Increased macrolide resistance of Mycoplasma pneumoniae in pediatric patients with community-acquired pneumonia. Antimicrob Agents Chemother 2008;52:348-50.

3. Chironna M, Sallustio A, Esposito S, et al. Emergence of macrolide-resistant strains during an outbreak of Mycoplasma pneumoniae infections in children. J Antimicrob Chemother 2011;66:734-7.

4. Waites KB, Ratliff A, Crabb DM, et al. MacrolideResistant Mycoplasma pneumoniae in the United States as Determined from a National Surveillance Program. J Clin Microbiol 2019;57:e00968-19.

5. Zhao F, Li J, Liu J, et al. Antimicrobial susceptibility and molecular characteristics of Mycoplasma pneumoniae isolates across different regions of China. Antimicrob Resist Infect Control 2019;8:143.

6. Liu Y, Ye X, Zhang H, et al. Characterization of macrolide resistance in Mycoplasma pneumoniae isolated from children in Shanghai, China. Diagn Microbiol Infect Dis 2010;67:355-8.

7. Kawai Y, Miyashita N, Kubo M, et al. Therapeutic efficacy of macrolides, minocycline, and tosufloxacin against macrolide-resistant Mycoplasma pneumoniae pneumonia in pediatric patients. Antimicrob Agents Chemother 2013;57:2252-8.

8. Okada T, Morozumi M, Tajima T, et al. Rapid effectiveness of minocycline or doxycycline against macrolide-resistant Mycoplasma pneumoniae infection in a 2011 outbreak among Japanese children. Clin Infect Dis 2012;55:1642-9.

9. Ding Y, Chu C, Li Y, et al. High expression of HMGB1 in children with refractory Mycoplasma pneumoniae pneumonia. BMC Infect Dis 2018;18:439.

10. Yang HJ, Song DJ, Shim JY. Mechanism of resistance acquisition and treatment of macrolide-resistant Mycoplasma pneumoniae pneumonia in children. Korean J Pediatr 2017;60:167-74.

11. Komatsu H, Tsunoda T, Inui A, et al. Characteristics of hospitalized children infected with macrolide-resistant Mycoplasma pneumoniae. Braz J Infect Dis 2014;18:294-9.

12. Uehara S, Sunakawa K, Eguchi H, et al. Japanese
Guidelines for the Management of Respiratory Infectious Diseases in Children 2007 with focus on pneumonia. Pediatr Int 2011;53:264-76.

13. McIntosh K. Community-acquired pneumonia in children. N Engl J Med 2002;346:429-37.

14. Ha SG, Oh KJ, Ko KP, et al. Therapeutic Efficacy and Safety of Prolonged Macrolide, Corticosteroid, Doxycycline, and Levofloxacin against MacrolideUnresponsive Mycoplasma pneumoniae Pneumonia in Children. J Korean Med Sci 2018;33:e268.

15. The Subspecialty Group of Respiratory Diseases. Expert consensus on diagnosis and treatment of mycoplasma pneumoniae pneumonia in children (2015). Chin J Appl Clin Pediatr 2015;30:1304-8.

16. Chen J, Ji F, Yin Y, et al. Time to Mycoplasma Pneumoniae RNA Clearance for Wheezy vs. Non-Wheezy Young Children with Community-Acquired Pneumonia. J Trop Pediatr 2021;67:fmaa109.

17. Socan M, Kosmelj K, Marinic-Fiser N, et al. A prediction model for community-acquired Chlamydia pneumoniae pneumonia in hospitalized patients. Infection 2004;32:204-9.

18. Li HN, Zhao X, Zha YJ, et al. miR-146a-5p suppresses ATP-binding cassette subfamily $\mathrm{G}$ member 1 dysregulation in patients with refractory Mycoplasma pneumoniae via interleukin 1 receptor-associated kinase 1 downregulation. Int J Mol Med 2019;44:2003-14.

19. Zhou Y, Wang J, Chen W, et al. Impact of viral coinfection and macrolide-resistant mycoplasma infection in children with refractory Mycoplasma pneumoniae pneumonia. BMC Infect Dis 2020;20:633.

20. Yan Y, Wei Y, Jiang W, et al. The clinical characteristics of corticosteroid-resistant refractory Mycoplasma Pneumoniae pneumonia in children. Sci Rep 2016;6:39929.

21. Morozumi M, Okada T, Tajima T, et al. Killing kinetics of minocycline, doxycycline and tosufloxacin against macrolide-resistant Mycoplasma pneumoniae. Int J Antimicrob Agents 2017;50:255-7.

22. Guo DX, Hu WJ, Wei R, et al. Epidemiology and mechanism of drug resistance of Mycoplasma pneumoniae in Beijing, China: A multicenter study. Bosn J Basic Med Sci 2019;19:288-96.

23. Garrido-Mesa N, Zarzuelo A, Gálvez J. Minocycline: far beyond an antibiotic. Br J Pharmacol 2013;169:337-52.

24. Cheng S, Lin J, Zheng X, et al. Development and validation of a simple-to-use nomogram for predicting refractory Mycoplasma pneumoniae pneumonia in children. Pediatr Pulmonol 2020;55:968-74. 
25. Miyashita N, Akaike H, Teranishi H, et al. Macrolideresistant Mycoplasma pneumoniae pneumonia in adolescents and adults: clinical findings, drug susceptibility, and therapeutic efficacy. Antimicrob Agents Chemother 2013;57:5181-5.

26. Ishiguro N, Koseki N, Kaiho M, et al. Therapeutic efficacy of azithromycin, clarithromycin, minocycline and tosufloxacin against macrolide-resistant and macrolidesensitive Mycoplasma pneumoniae pneumonia in pediatric patients. PLoS One 2017;12:e0173635.

27. Nakazawa S, Oka S, Sato H, et al. Effects of dry syrup minocycline (Minomycin 'Lederle') in the pediatric field. Jpn J Antibiot 1972;25:288-94.

Cite this article as: Chen J, Qi X, Yin Y, Zhang L, Zhang J, Yuan S. Effects of minocycline on macrolide-unresponsive Mycoplasma pneumoniae pneumonia in children: a singlecenter retrospective study. Transl Pediatr 2021;10(11):29973004. doi: $10.21037 / \mathrm{tp}-21-356$ 